\title{
openheart Health system barriers and facilitators to medication adherence for the secondary prevention of cardiovascular disease: a systematic review
}

Amitava Banerjee, ${ }^{1}$ Shweta Khandelwal, ${ }^{2}$ Lavanya Nambiar, ${ }^{2}$ Malvika Saxena, ${ }^{2}$ Victoria Peck, ${ }^{3}$ Mohammed Moniruzzaman, ${ }^{4}$ Jose Rocha Faria Neto, ${ }^{5}$ Katherine Curi Quinto, ${ }^{6}$ Andrew Smyth, ${ }^{3}$ Darryl Leong, ${ }^{3}$ José Pablo Werba ${ }^{7}$

To cite: Banerjee A, Khandelwal S, Nambiar L, et al. Health system barriers and facilitators to medication adherence for the secondary prevention of cardiovascular disease: a systematic review. Open Heart 2016;3: 0000438 doi:10.1136/openhrt-2016000438

- Additional material is available. To view please visit the journal (http://dx.doi.org/ 10.1136/openhrt-2016000438).

Received 17 March 2016 Revised 12 August 2016 Accepted 22 August 2016

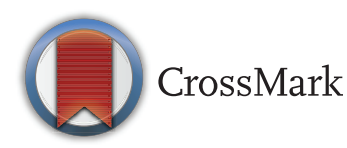

For numbered affiliations see end of article.

Correspondence to Dr Amitava Banerjee; ami.banerjee@ucl.ac.uk

\section{ABSTRACT}

Background: Secondary prevention is cost-effective for cardiovascular disease (CVD), but uptake is suboptimal. Understanding barriers and facilitators to adherence to secondary prevention for CVD at multiple health system levels may inform policy.

Objectives: To conduct a systematic review of barriers and facilitators to adherence/persistence to secondary CVD prevention medications at health system level.

Methods: Included studies reported effects of health system level factors on adherence/persistence to secondary prevention medications for CVD (coronary artery or cerebrovascular disease). Studies considered at least one of $\beta$ blockers, statins, angiotensin-renin system blockers and aspirin. Relevant databases were searched from 1 January 1966 until 1 October 2015. Full texts were screened for inclusion by 2 independent reviewers.

Results: Of 2246 screened articles, 25 studies were included (12 trials, 11 cohort studies, 1 cross-sectional study and 1 case-control study) with 132140 individuals overall (smallest $n=30$, largest $n=63301$ ). 3 studies included upper middle-income countries, 1 included a low middle-income country and 21 (84\%) included high-income countries ( 9 in the USA). Studies concerned established CVD $(n=4)$, cerebrovascular disease $(n=7)$ and coronary heart disease $(n=14)$. Three studies considered persistence and adherence. Quantity and quality of evidence was limited for adherence, persistence and across drug classes. Studies were concerned with governance and delivery $(n=19$, including 4 trials of fixed-dose combination therapy, FDC), intellectual resources $(n=1)$, human resources $(n=1)$ and health system financing $(n=4)$. Full prescription coverage, reduced copayments, FDC and counselling were facilitators associated with higher adherence. Conclusions: High-quality evidence on health system barriers and facilitators to adherence to secondary prevention medications for CVD is lacking, especially for low-income settings. Full prescription coverage, reduced copayments, FDC and counselling may be effective in improving adherence and are priorities for further research.

\section{KEY QUESTIONS}

What is already known about this subject?

- Despite proven cost-effectiveness of medications for secondary prevention of cardiovascular disease, adherence is suboptimal.

What does this study add?

- The barriers and facilitators to medication adherence at health system level are poorly characterised, particularly in low-income settings. Full prescription coverage, reduced copayments, fixed-dose combination (FDC) therapy and counselling are effective on the basis of existing data, but further research is needed.

How might this impact on clinical practice?

- At health system level, finance mechanisms, FDC therapy and counselling should be prioritised in research and implementation to promote medication adherence in individuals with cardiovascular disease.

\section{INTRODUCTION}

Cardiovascular disease (CVD) is the leading threat to global health, whether measured by mortality, morbidity or economic cost. ${ }^{1}$ The greatest burden of CVD is in low and middle-income countries, with crippling macroeconomic effects. ${ }^{1-4}$ Health policy focussing on CVD has become a priority to policymakers, scientists, health professionals and patients. ${ }^{5-7}$

Secondary prevention represents a crucial and cost-effective component of the response due to the high absolute risk of recurrent cardiovascular events in individuals with established CVD. ${ }^{8}$ Above and beyond effective lifestyle interventions (smoking cessation, physical activity and appropriate diet), there is high-quality evidence that drug therapy with aspirin, statins, angiotensin-converting 
enzyme inhibitors (ACEI) or angiotensin-receptor blockers (ARBs) and $\beta$ blockers is effective in reducing morbidity and mortality from CVD in individuals with pre-existing CVD. ${ }^{9}$ Although these medications are offpatent and should be available at low cost, uptake is suboptimal. ${ }^{10}$ The problem is heightened in the poorest settings, but there are still significant gaps even in affluent countries. ${ }^{11} 12$

Obstacles to evidence-based secondary CVD prevention are context-specific, and differences in healthcare systems between countries of varying incomes are important contributors to differences in health outcomes. ${ }^{10}$ For example, the Population Urban Rural Epidemiology (PURE) study demonstrated higher CVD mortality in low-income countries than in high-income countries, despite a lower risk factor burden in the former. ${ }^{13}$ However, there has not been comprehensive evaluation of barriers and facilitators to medication adherence for CVD secondary prevention at multiple health system levels and in diverse financial and sociocultural settings, which would inform health policy. The objective of this systematic review was therefore to identify health system features, programmes or strategies which act as barriers or facilitators to adherence to evidence-supported medications for CVD secondary prevention.

\section{METHODS}

A protocol for this study has been published (PROSPERO 2015: CRD42015019079). We used an established framework ${ }^{14}$ to illustrate the health system and its elements (figure 1). This conceptual framework consists of health system 'inputs' (which include physical, human, intellectual and social resources) plus components and characteristics of delivery, governance and financing. ${ }^{14}$

\section{Inclusion criteria}

We included quantitative and qualitative studies reporting associations of local, national, regional or international health system level factors, interventions, policies or programmes with adherence to medications for the secondary prevention of CVD (coronary artery or cerebrovascular disease) until 1 October 2015 with no language restrictions. Included studies had analyses of barriers and facilitators to adherence or persistence to at least one of $\beta$ blockers, statins, angiotensin-renin system blockers and aspirin. Any outcome measures of adherence ("the extent to which a patient acts in accordance with the prescribed interval and dose of a dosing regimen") or persistence ("the duration of time from initiation to discontinuation of therapy") ${ }^{15}$ were considered. We excluded studies reporting prescription and usage alone.

\section{Search strategy}

Our search included MEDLINE, EMbase, Cochrane Library, Psychinfo, Health Systems Evidence, Health Management Information Consortium (HMIC), Latin American and Caribbean Health Sciences Literature (LILACS), Africa-Wide Information and Google Scholar. We also searched conference proceedings and reference lists of relevant research articles. We also consulted experts in health policy regarding access to medicines. Our search terms and actual strategy are further detailed in the online supplementary appendix.

\section{Study selection}

Each title and/or abstract identified by the search strategy was independently reviewed for potential eligibility by two investigators (from AB, MM, KCQ LN, MS, VP, JPW, JRFN). Full texts were obtained and further screened for inclusion by two reviewers. Disagreements were resolved by a third reviewer.

\section{Data extraction}

Prior to data extraction, a validation exercise was conducted to ensure consistency with respect to data extracted from five articles. Data were extracted from each study on study design, setting, methods and outcomes; health system domains investigated, health system barriers or facilitators. Data were extracted according to a 'health system framework': ${ }^{14}$ (1) health

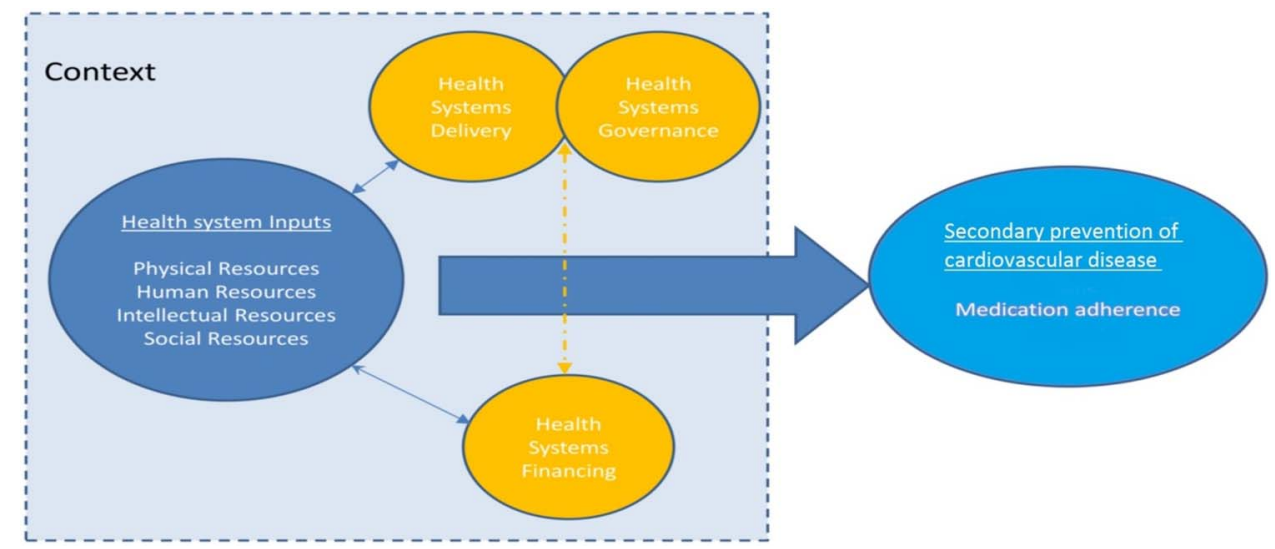

Figure 1 Schematic diagram of health systems conceptual framework (from Maimaris et al ${ }^{14}$ with permission from PLoS). 
systems delivery; (2) health systems governance; (3) health systems financing; (4) health systems inputs: physical, human, intellectual and social resources and (5) outcomes for secondary prevention medications of CVD: adherence and persistence. Two reviewers (AB and JPW) checked consistency of inclusion criteria and data extraction across all included studies. At the data extraction stage, there was disagreement in $1 / 25$ studies (4.0\%), which was resolved by discussion between $\mathrm{AB}$ and JPW.

\section{Assessment of the risk of bias}

Included studies were independently assessed for risk of bias by two reviewers using a framework previously used for observational study designs in similar systematic reviews: selection bias, information bias (differential misclassification and non-differential misclassification) and confounding. ${ }^{14}$ Risk of bias was assessed as either low, unclear or high for each domain and overall. The Cochrane tool for reporting of bias was used for clinical trials (see online supplementary appendix 2). Disagreements in classification of bias and data extraction were resolved by a third reviewer $(\mathrm{AB}$ or JPW). Publication bias was assessed by funnel plot analysis. Conflicts of interest were not assessed.

\section{Data synthesis and analysis}

Studies were categorised according to health system domain and setting. ORs were recorded, if reported, as "OR, 95\% confidence interval; $p$ value". Where possible,
OR or RR was calculated from available data, if not reported. Reporting complied with standards in 'Preferred Reporting Items for Systematic Reviews and Meta-Analyses' (PRISMA). ${ }^{16}$ Meta-analysis was not possible due to heterogeneity of included studies across multiple domains, including study populations, study designs, varying definitions of exposures and outcomes and analytical strategies.

\section{RESULTS}

Figure 2 shows the PRISMA flow chart. A total 2246 articles were screened by title and abstract. Full texts of 269 of the 2246 articles were assessed for eligibility. Twenty-five quantitative and no qualitative studies met eligibility criteria for this review. Table 1 shows characteristics of included studies by domains of the conceptual framework described by Maimaris $e t a l .{ }^{14}$ Full details are in table 2. Online supplementary appendix tables 1a-c summarise included studies by adherence, persistence and drug class, respectively. Tables 3 and 4 show risk of bias assessment for included trials and observational studies, respectively.

\section{Included study characteristics}

In total, there were 132140 individuals in the 25 studies, with the smallest study of $\mathrm{n}=30^{17}$ and the largest study of $\mathrm{n}=63301 .{ }^{18}$ Of the 25 included studies, 11 were randomised controlled trials (RCTs) $;^{17}{ }^{19-28} 1$ was a nonrandomised trial, ${ }^{29} 11$ were cohort studies, ${ }^{18}{ }^{30-39} 1$ was

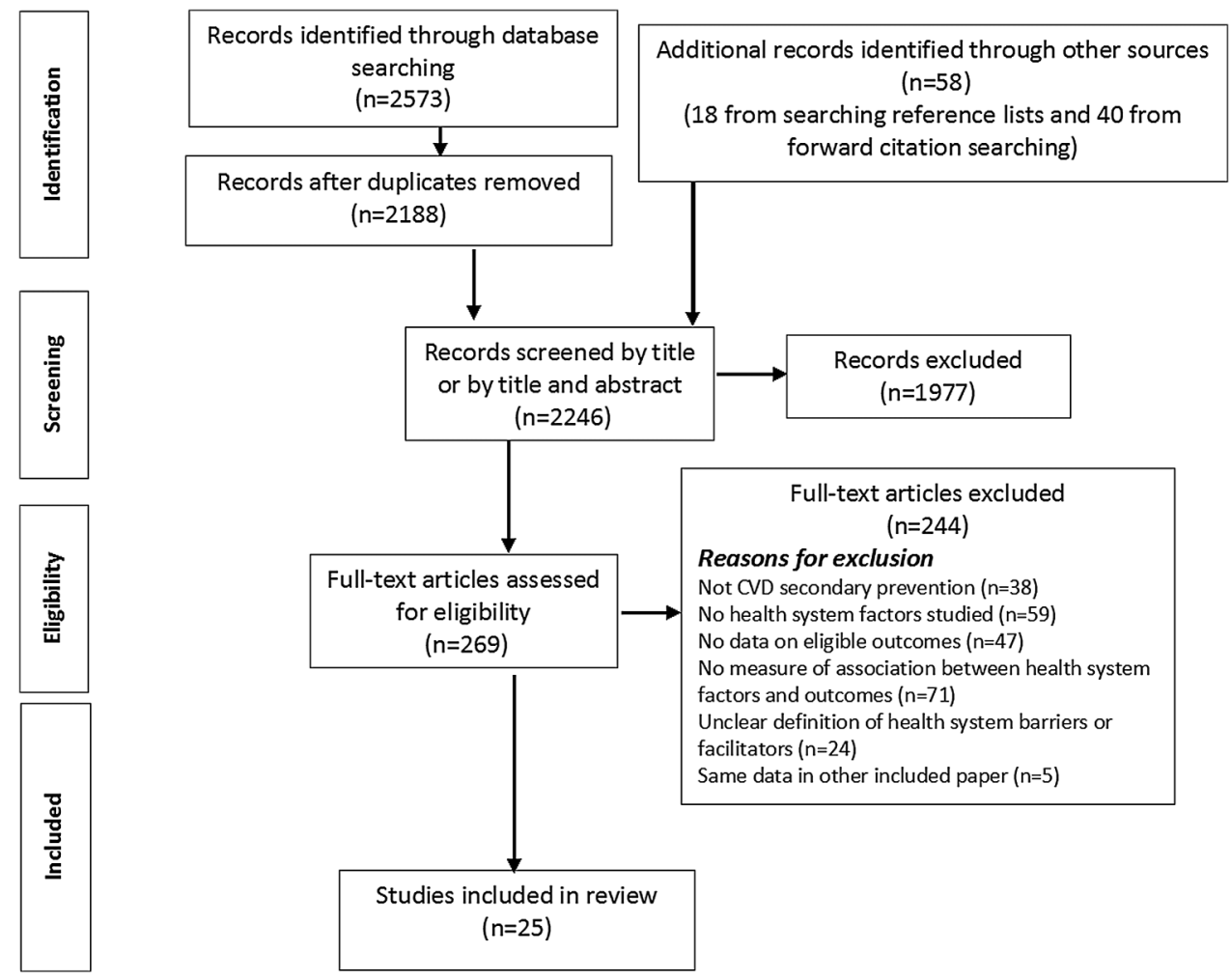

Figure 2 PRISMA flow chart. CVD, cardiovascular disease. PRISMA, Preferred Reporting Items for Systematic Reviews and Meta-Analyses. 
Table 1 Health system arrangements investigated by included studies, classified by health system domain

\begin{tabular}{|c|c|c|c|c|}
\hline $\begin{array}{l}\text { Health system } \\
\text { framework } \\
\text { domain }\end{array}$ & $\begin{array}{l}\text { Health system } \\
\text { factor being } \\
\text { investigated }\end{array}$ & $\begin{array}{l}\text { Number of } \\
\text { studies }\end{array}$ & $\begin{array}{l}\text { Number of studies and } \\
\text { study designs }\end{array}$ & Setting of studies (countries) \\
\hline \multirow[t]{15}{*}{$\begin{array}{l}\text { Governance and } \\
\text { delivery }\end{array}$} & $\begin{array}{l}\text { Physician-led } \\
\text { education }\end{array}$ & 1 & RCT (1) & UK $(1)^{21}$ \\
\hline & Nurse-led education & 3 & $\mathrm{RCT}(2)$ & Denmark (1), ${ }^{22}$ UK $(1)^{23}$ \\
\hline & & & Cohort (1) & USA/Canada $(1)^{39}$ \\
\hline & $\begin{array}{l}\text { Pharmacy-led } \\
\text { education }\end{array}$ & 2 & $\mathrm{RCT}(1)$ & USA $(1)^{17}$ \\
\hline & & & Non-randomised trial (1) & Germany $(1)^{29}$ \\
\hline & $\begin{array}{l}\text { Comprehensive } \\
\text { education } \\
\text { programme }\end{array}$ & 2 & $\mathrm{RCT}(1)$ & Turkey $(1)^{24}$ \\
\hline & & & Cohort (1) & France $(1)^{36}$ \\
\hline & $\begin{array}{l}\text { Hospital-level quality } \\
\text { improvement }\end{array}$ & 4 & $\mathrm{RCT}(1)$ & USA $(1)^{19}$ \\
\hline & & & Cohort (2) & USA $(1)^{30}$ Canada $(1)^{31}$ \\
\hline & & & Case-control (1) & USA $(1)^{41}$ \\
\hline & Routine place of care & 1 & Cohort (1) & Sweden $(1)^{32}$ \\
\hline & $\begin{array}{l}\text { Generic versus } \\
\text { branded drugs }\end{array}$ & 1 & Cohort (1) & USA $(1)^{37}$ \\
\hline & $\begin{array}{l}\text { Complexity of } \\
\text { treatment regimen }\end{array}$ & 1 & Cross-sectional (1) & $\begin{array}{l}\text { Argentina/Brazil/taly/Paraguay/ } \\
\text { Spain }(1)^{25}\end{array}$ \\
\hline & FDC & 4 & $\mathrm{RCT}(4)$ & $\begin{array}{l}\text { India/Europe }(1),{ }^{20} \text { Australia/New } \\
\text { Zealand }(1),{ }^{26} \text { New Zealand }(1),{ }^{28} \\
\text { Argentina/Brazil/Italy/Paraguay/ } \\
\text { Spain }(1)^{25}\end{array}$ \\
\hline & $\begin{array}{l}\text { All governance and } \\
\text { delivery studies }\end{array}$ & 19 & $\begin{array}{l}R C T(10), \text { non-randomised } \\
\text { trial (1), cohort (6), } \\
\text { cross-sectional (1), case- } \\
\text { control (1) }\end{array}$ & $\begin{array}{l}\text { USA (5), Canada (1), USA/Canada } \\
\text { (1), UK(2), Germany (1), Sweden } \\
\text { (1), Turkey (1), France (1), } \\
\text { Denmark (1), India/Europe (1), } \\
\text { Australia/New Zealand (1), New } \\
\text { Zealand (1), Argentina/Brazil/Italy/ } \\
\text { Paraguay/Spain (2) }\end{array}$ \\
\hline \multirow[t]{2}{*}{$\begin{array}{l}\text { Human } \\
\text { resources }\end{array}$} & $\begin{array}{l}\text { Undergraduate } \\
\text { training of physicians }\end{array}$ & 1 & Cohort (1) & Canada $(1)^{18}$ \\
\hline & $\begin{array}{l}\text { All human resources } \\
\text { studies }\end{array}$ & 1 & Cohort (1) & Canada (1) \\
\hline \multirow[t]{2}{*}{$\begin{array}{l}\text { Intellectual } \\
\text { resources }\end{array}$} & $\begin{array}{l}\text { Education of } \\
\text { physicians }\end{array}$ & 1 & Cohort (1) & Israel $(1)^{33}$ \\
\hline & $\begin{array}{l}\text { All intellectual } \\
\text { resources studies }\end{array}$ & 1 & Cohort (1) & Israel (1) \\
\hline \multirow[t]{4}{*}{$\begin{array}{l}\text { Health system } \\
\text { financing }\end{array}$} & $\begin{array}{l}\text { Copayments for } \\
\text { medical care }\end{array}$ & 2 & Cohort (2) & USA (1), ${ }^{35}$ Austria $(1)^{34}$ \\
\hline & $\begin{array}{l}\text { Insurance and } \\
\text { prescription cost } \\
\text { assistance }\end{array}$ & 2 & Cohort (1) & USA $(1)^{38}$ \\
\hline & & & $\mathrm{RCT}(1)$ & USA $(1)^{27}$ \\
\hline & All financing studies & 4 & RCT (1), cohort (3) & USA (3), Austria (1) \\
\hline $\begin{array}{l}\text { Physical } \\
\text { resources }\end{array}$ & $\begin{array}{l}\text { All physical } \\
\text { resources studies }\end{array}$ & 0 & & \\
\hline Social resources & $\begin{array}{l}\text { All social resources } \\
\text { studies }\end{array}$ & 0 & & \\
\hline
\end{tabular}

cross-sectional $^{25}$ and 1 was case-control. ${ }^{40}$ Other than 3 studies including upper middle-income countries ${ }^{24} 25$ and one including a low middle-income country, ${ }^{20} 21$ of the 25 studies $(84 \%)$ were conducted in countries classified as high-income countries, 9 of which were in the USA. ${ }^{17} 19273035{ }^{37-40}$ Studies included individuals with cerebrovascular disease $(\mathrm{n}=7),{ }^{19} 21-23293032$ established CVD or an estimated 5-year CVD risk of $\geq 15 \%$ 


\begin{tabular}{|c|c|c|c|c|c|c|c|}
\hline $\begin{array}{l}\text { Barriers/ } \\
\text { facilitators }\end{array}$ & $\begin{array}{l}\text { Study (author, } \\
\text { year, setting) }\end{array}$ & Context & Study design & $\begin{array}{l}\text { Sample } \\
\text { size }\end{array}$ & Study details & Outcome & $\begin{array}{l}\text { Relevant findings }(95 \% \\
\text { Cls given where available } \\
\text { and in italics when } \\
p<0.05)\end{array}$ \\
\hline \multirow[t]{4}{*}{$\begin{array}{l}\text { Patient } \\
\text { counselling }\end{array}$} & $\begin{array}{l}\text { O'Carroll, } 2013 \\
(\mathrm{UK})^{21}\end{array}$ & First stroke/TIA & $\mathrm{RCT}$ & 62 & $\begin{array}{l}\text { Intervention=physician-led } \\
\text { counselling sessions aimed at } \\
\text { increasing adherence }\end{array}$ & $\begin{array}{l}\text { Adherence to } \\
\text { antihypertensive } \\
\text { medication at } \\
3 \text { months } \\
\text { Electronic pill } \\
\text { count and } \\
\text { self-report }\end{array}$ & $\begin{array}{l}\text { Intervention versus control: } \\
\text { by electronic pill count, } \\
\text { percentage of doses taken } \\
\text { on schedule-96.8\% vs } \\
87.4 \%, \text { mean difference } \\
9.8 \%, 95 \% \mathrm{Cl} 0.2 \text { to } 16.2 ; \\
p=0.048 \\
\text { Self-report highly correlated } \\
\text { with electronic pill count }\end{array}$ \\
\hline & $\begin{array}{l}\text { Hornnes, } 2011 \\
\text { (Denmark) }^{22}\end{array}$ & Acute stroke/TIA & $\mathrm{RCT}$ & 349 & $\begin{array}{l}\text { Intervention=four home visits } \\
\text { by a nurse with individually } \\
\text { tailored counselling on a } \\
\text { healthy lifestyle }\end{array}$ & $\begin{array}{l}\text { Adherence to } \\
\text { antihypertensive } \\
\text { therapy at } 1 \text { year } \\
\text { Self-report }\end{array}$ & $\begin{array}{l}\text { Intervention versus control: } \\
98 \% \text { vs } 99 \% \text {, OR } 0.88 \\
95 \% \text { Cl } 0.54 \text { to } 1.44 ; \\
p=0.50\end{array}$ \\
\hline & $\begin{array}{l}\text { Maron, } 2010 \\
\text { (USA and } \\
\text { Canada) }^{39}\end{array}$ & Stable CHD & Prospective cohort & 2287 & $\begin{array}{l}\text { Nurse-led case management } \\
\text { nested in the Clinical } \\
\text { Outcomes Utilizing } \\
\text { Revascularization and } \\
\text { Aggressive Drug Evaluation } \\
\text { (COURAGE) Trial. CVD drugs } \\
\text { provided at no cost }\end{array}$ & $\begin{array}{l}\text { Adherence and } \\
\text { persistence to } 4 \mathrm{D} \\
\text { at } 5 \text { years } \\
\text { Self-report }\end{array}$ & $\begin{array}{l}\text { Persistence increased from } \\
\text { baseline to } 5 \text { years as } \\
\text { follows: antiplatelets } 87 \% \text { to } \\
96 \%,(O R 3.58,95 \% \mathrm{Cl} \\
2.48 \text { to } 5.18) ; \beta \text { blockers } \\
69 \% \text { to } 85 \% \text { (OR 2.54, } 2.06 \\
\text { to } 3.15) ; \text { ARBs } 46 \% \text { to } 72 \% \\
\text { (OR 3.02, } 2.53 \text { to } 3.60) \text {, } \\
\text { statins } 64 \% \text { to } 93 \%(O R \\
7.51,5.67 \text { to } 9.94), 4 D 28 \% \\
\text { to } 53 \%(O R 2.90,95 \% C l \\
2.44 \text { to } 3.43)(a l l p<0.001) \text {. } \\
\text { Adherence was } 97 \% \text { at } \\
6 \text { months and } 95 \% \text { at } \\
5 \text { years }\end{array}$ \\
\hline & $\begin{array}{l}\text { McManus, } \\
2009 \text { (UK) }^{23}\end{array}$ & Stroke in hospital & $\mathrm{RCT}$ & 102 & $\begin{array}{l}\text { Intervention }=3 \text { months } \\
\text { nurse-led health counselling } \\
\text { with written and verbal } \\
\text { information on lifestyle, and } \\
\text { check of medication } \\
\text { concordance }\end{array}$ & $\begin{array}{l}\text { Adherence and } \\
\text { persistence to } 4 \mathrm{D} \\
\text { at } 3 \text { years } \\
\text { Self-report }\end{array}$ & $\begin{array}{l}\text { Persistence: } 95 \% \text { vs } 89 \% \text {, } \\
\text { OR } 3.00,0.57 \text { to } 15.7 \\
\text { ( } p=0.19 \text { ) for antiplatelets } \\
97 \% \text { vs } 95 \% \text {, OR } 1.02,0.55 \\
\text { to } 1.91(p=0.95) \text { for } \\
\text { antihypertensives } \\
88 \% \text { vs } 89 \% \text {, OR } 1.03,0.25 \\
\text { to } 4.14(p=0.97) \text { for statins } \\
\text { Adherence to } 4 D: 78 \% \text { vs } \\
92 \%, \text { OR } 0.30,0.07 \text { to } 1.24 \\
(p=0.10)\end{array}$ \\
\hline
\end{tabular}




\begin{tabular}{|c|c|c|c|c|c|c|c|}
\hline $\begin{array}{l}\text { Barriers/ } \\
\text { facilitators }\end{array}$ & $\begin{array}{l}\text { Study (author, } \\
\text { year, setting) }\end{array}$ & Context & Study design & $\begin{array}{l}\text { Sample } \\
\text { size }\end{array}$ & Study details & Outcome & $\begin{array}{l}\text { Relevant findings }(95 \% \\
\text { Cls given where available } \\
\text { and in italics when } \\
p<0.05)\end{array}$ \\
\hline & $\begin{array}{l}\text { Faulkner, } 2000 \\
(\text { USA })^{17}\end{array}$ & CABG & $\mathrm{RCT}$ & 30 & $\begin{array}{l}\text { Intervention=weekly } \\
\text { pharmacist-led telephone } \\
\text { contact for } 12 \text { weeks }\end{array}$ & $\begin{array}{l}\text { Adherence to } \\
\text { lovastatin at } \\
1 \text { year and } \\
2 \text { years } \\
\text { Prescription fill } \\
\text { rate }\end{array}$ & $\begin{array}{l}\text { Intervention versus control: } \\
67 \% \text { vs } 33 \% ; p<0.05 \text { at } \\
1 \text { year and } 60 \% \text { vs } 27 \% ; \\
p<0.05 \text { at } 2 \text { years }\left(\chi^{2} \text { test }\right. \\
\text { reported) } \\
\text { At } 1 \text { year, OR } 4.00,0.88 \text { to } \\
18.26 ; p=0.07, \text { and at } \\
2 \text { years, OR } 4.13,0.88 \text { to } \\
19.27 ; p=0.07\end{array}$ \\
\hline & $\begin{array}{l}\text { Hohmann, } \\
2009 \\
\text { (Germany) }^{29}\end{array}$ & $\begin{array}{l}\text { Ischaemic stroke/ } \\
\text { TIA in hospital }\end{array}$ & $\begin{array}{l}\text { Non-randomised, } \\
\text { controlled } \\
\text { intervention trial }\end{array}$ & 255 & $\begin{array}{l}\text { Intervention=hospital } \\
\text { pharmacist counselling before } \\
\text { discharge and plan for } \\
\text { outpatient care plus } \\
\text { counselling by community } \\
\text { pharmacists }\end{array}$ & $\begin{array}{l}\text { Persistence to } \\
\text { aspirin and } \\
\text { clopidogrel at } \\
1 \text { year } \\
\text { Self-reported and } \\
\text { GP-reported }\end{array}$ & $\begin{array}{l}\text { Intervention: } 38.7 \% \text { vs } \\
32.7 \%, \text { OR } 1.30,0.73 \text { to } \\
2.31 ; p=0.37 \text { for aspirin and } \\
26.7 \% \text { and } 30.1 \%, O R \\
0.85,0.46 \text { to } 1.57 ; p=0.60 \\
\text { for clopidogrel }\end{array}$ \\
\hline & $\begin{array}{l}\text { Lafitte, } 2009 \\
\text { (France) }^{36}\end{array}$ & ACS in hospital & Prospective cohort & 660 & $\begin{array}{l}3 \text { months after discharge for } \\
\text { ACS, consecutive patients } \\
\text { were invited to join a } \\
\text { comprehensive risk factor } \\
\text { management programme }\end{array}$ & $\begin{array}{l}\text { Persistence to 4D } \\
\text { at } 20 \text { months } \\
\text { (mean follow-up) } \\
\text { Self-report }\end{array}$ & $\begin{array}{l}\text { At follow-up and baseline, } \\
\text { respectively (no control } \\
\text { group reported): } 86 \% \text { vs } \\
98 \% \text { for } \beta \text { blocker or a } \\
\text { calcium antagonist, } 88 \% \text { vs } \\
94 \% \text { for statin, } 96 \% \text { vs } \\
100 \% \text { for antiplatelet, } 62 \% \\
\text { vs } 82 \% \text { for ACEI/ARB, } 76 \% \\
\text { vs } 92 \% \text { for } 4 D\end{array}$ \\
\hline & $\begin{array}{l}\text { Yilmaz, } 2005 \\
\text { (Turkey) }^{24}\end{array}$ & $\begin{array}{l}\text { Secondary } \\
\text { prevention in } \\
\text { hospital }\end{array}$ & RCT & 202 & $\begin{array}{l}\text { Intervention=counselling } \\
\text { regarding efficacy, } \\
\text { pharmacokinetic profile, and } \\
\text { side effects of ongoing statins }\end{array}$ & $\begin{array}{l}\text { Persistence to } \\
\text { statin therapy at } \\
15 \text { months } \\
\text { (median } \\
\text { follow-up) } \\
\text { Self-report }\end{array}$ & $\begin{array}{l}62.7 \% \text { vs } 46 \% ; O R=1.98 \\
1.13 \text { to } 3.47 ; p=0.017\end{array}$ \\
\hline $\begin{array}{l}\text { Hospital quality } \\
\text { improvement } \\
\text { programmes }\end{array}$ & $\begin{array}{l}\text { Bushnell, } 2011 \\
(\text { USA) }\end{array}$ & $\begin{array}{l}\text { Ischaemic stroke/ } \\
\text { TIA in hospital }\end{array}$ & $\begin{array}{l}\text { Retrospective } \\
\text { cohort }\end{array}$ & 2457 & $\begin{array}{l}\text { Guideline implementation in } \\
\text { the Adherence eValuation After } \\
\text { Ischemic stroke-Longitudinal } \\
\text { (AVAIL) Registry in a sample of } \\
\text { hospitals participating in the } \\
\text { Get With The Guidelines- } \\
\text { Stroke program }\end{array}$ & $\begin{array}{l}\text { Persistence and } \\
\text { adherence to } 4 D \\
\text { at } 1 \text { year } \\
\text { Self-report }\end{array}$ & $\begin{array}{l}\text { Persistence and adherence } \\
\text { associated with: number of } \\
\text { medications prescribed at } \\
\text { discharge (OR=1.08, } 1.04 \\
\text { to } 1.11 ; p<0.001 \text { per } 1 \\
\text { decrease); and follow-up } \\
\text { appointment with GP } \\
\text { (OR=1.72, } 1.12 \text { to } 2.52 ; \\
p=.0 .006)\end{array}$ \\
\hline
\end{tabular}

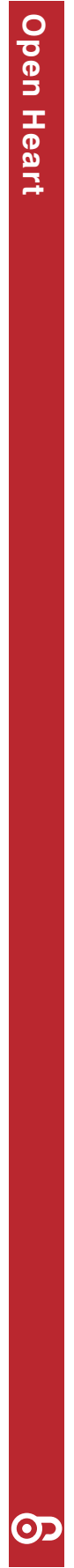

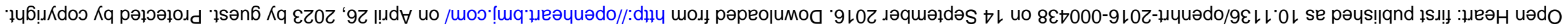




\begin{tabular}{|c|c|c|c|c|c|c|c|}
\hline $\begin{array}{l}\text { Barriers/ } \\
\text { facilitators }\end{array}$ & $\begin{array}{l}\text { Study (author, } \\
\text { year, setting) }\end{array}$ & Context & Study design & $\begin{array}{l}\text { Sample } \\
\text { size }\end{array}$ & Study details & Outcome & $\begin{array}{l}\text { Relevant findings }(95 \% \\
\text { Cls given where available } \\
\text { and in italics when } \\
p<0.05)\end{array}$ \\
\hline & $\begin{array}{l}\text { Jackevicius, } \\
2008 \\
\text { (Canada) }^{31}\end{array}$ & AMI in hospital & $\begin{array}{l}\text { Retrospective } \\
\text { cohort }\end{array}$ & 4591 & $\begin{array}{l}\text { Quality improvement of care in } \\
\text { the Enhanced Feedback for } \\
\text { Effective Cardiac Treatment } \\
\text { (EFFECT) study registry in } \\
\text { Ontario }\end{array}$ & $\begin{array}{l}\text { Adherence to } 4 \mathrm{D} \\
\text { at } 120 \text { days } \\
\text { Prescription fill } \\
\text { rate }\end{array}$ & $\begin{array}{l}\text { Predischarge medication } \\
\text { counselling: OR } 1.61,1.26 \\
\text { to } 2.04 ; p=0.0001 \\
\text { Cardiologist (vs GP) as } \\
\text { doctor responsible for } \\
\text { patient's care: OR } 1.80 \text {, } \\
1.34 \text { to } 2.43 ; p=0.0001 \text {. } \\
\text { Teaching versus other } \\
\text { hospital: OR } 1.35,0.93 \text { to } \\
1.97 ; p=0.11\end{array}$ \\
\hline & $\begin{array}{l}\text { Johnston, } 2010 \\
(\text { USA })^{19}\end{array}$ & $\begin{array}{l}\text { Ischaemic stroke } \\
\text { in hospital }\end{array}$ & $\mathrm{RCT}$ & 3361 & $\begin{array}{l}\text { Intervention: assistance in the } \\
\text { development and } \\
\text { implementation of standardised } \\
\text { stroke discharge orders }\end{array}$ & $\begin{array}{l}\text { Adherence to } \\
\text { statin at } 6 \text { months } \\
\text { Prescription fill } \\
\text { rate }\end{array}$ & $\begin{array}{l}\text { Intervention versus } \\
\text { non-intervention hospitals, } \\
\text { At hospital level: OR, } 1.26 \text {; } \\
0.70 \text { to } 2.30 ; p=0.36 \text {. } \\
\text { At individual level: } O R \text {, } \\
1.29 ; 1.04 \text { to } 1.60 ; p=0.02\end{array}$ \\
\hline & $\begin{array}{l}\text { Khanderia, } \\
2005 \text { (USA) }^{40}\end{array}$ & CABG in hospital & $\begin{array}{l}\text { Retrospective } \\
\text { case-control }\end{array}$ & 403 & $\begin{array}{l}\text { A physician education protocol } \\
\text { to implement statin in all } \\
\text { patients admitted for CABG }\end{array}$ & $\begin{array}{l}\text { Persistence to } \\
\text { statins at } 6 \\
\text { months } \\
\text { Self-report }\end{array}$ & $\begin{array}{l}\text { Intervention versus control: } \\
67 \% \text { vs } 58 \% \text {, OR } 1.49,0.88 \\
\text { to } 2.55 ; p=0.14\end{array}$ \\
\hline $\begin{array}{l}\text { Site of care and } \\
\text { home } \\
\text { circumstances } \\
\text { of patients }\end{array}$ & $\begin{array}{l}\text { Glader, } 2010 \\
\text { (Sweden) }^{32}\end{array}$ & $\begin{array}{l}\text { Acute stroke in } \\
\text { hospital }\end{array}$ & Prospective cohort & 21077 & $\begin{array}{l}\text { A 1-year cohort (September } \\
\text { 2005-August 2006) from the } \\
\text { Swedish Stroke Register }\end{array}$ & $\begin{array}{l}\text { Persistence with } \\
4 \mathrm{D} \text { at } 1 \text { year } \\
\text { Prescription fill } \\
\text { rate }\end{array}$ & $\begin{array}{l}\text { Institutional living correlated } \\
\text { with persistence for all drug } \\
\text { classes }(p=0.001) \text {. Stroke } \\
\text { unit care was associated } \\
\text { with persistence for statins } \\
\text { ( } p=0.007) \text {. } \\
\text { Support by next-of-kin } \\
\text { associated with persistence } \\
\text { for antihypertensives } \\
(p=0.001)\end{array}$ \\
\hline $\begin{array}{l}\text { Generic versus } \\
\text { branded drugs }\end{array}$ & $\begin{array}{l}\text { O'Brien, } 2015 \\
\text { (USA) }^{37}\end{array}$ & $\begin{array}{l}\text { NSTEMI in } \\
\text { hospital }\end{array}$ & $\begin{array}{l}\text { Retrospective } \\
\text { cohort }\end{array}$ & 1421 & $\begin{array}{l}\text { NSTEMI patients } \geq 65 \text { years old } \\
\text { discharged on a statin in } 2006 \\
\text { from USA hospitals }\end{array}$ & $\begin{array}{l}\text { Adherence to } \\
\text { statins at } 1 \text { year } \\
\text { Prescription refill } \\
\text { rate }\end{array}$ & $\begin{array}{l}\text { Generic versus brand } \\
\text { users: } 86.0 \%(I Q R=42.6- \\
97.2 \%) \text { vs } 84.1 \% \\
(I Q R=53.4-97.0 \%)) \\
(p=0.97)\end{array}$ \\
\hline
\end{tabular}




\begin{tabular}{|c|c|c|c|c|c|c|c|}
\hline $\begin{array}{l}\text { Barriers/ } \\
\text { facilitators }\end{array}$ & $\begin{array}{l}\text { Study (author, } \\
\text { year, setting) }\end{array}$ & Context & Study design & $\begin{array}{l}\text { Sample } \\
\text { size }\end{array}$ & Study details & Outcome & $\begin{array}{l}\text { Relevant findings ( } 95 \% \\
\text { Cls given where available } \\
\text { and in italics when } \\
p<0.05 \text { ) }\end{array}$ \\
\hline $\begin{array}{l}\text { Complexity of } \\
\text { treatment } \\
\text { regimen }\end{array}$ & $\begin{array}{l}\text { Castellano, } \\
2014 \\
\text { (Argentina, } \\
\text { Brazil, Italy, } \\
\text { Paraguay and } \\
\text { Spain) }\end{array}$ & $\begin{array}{l}\text { Aged }>40 \text { years } \\
\text { with AMI in last } \\
2 \text { years }\end{array}$ & $\begin{array}{l}\text { Cross-sectional } \\
\text { study }\end{array}$ & 2118 & $\begin{array}{l}\text { In a single visit, data was } \\
\text { gathered to estimate } \\
\text { prescription, adherence and } \\
\text { barriers to adherence for } \\
\text { aspirin, ACEls, } \beta \text { blockers and } \\
\text { statins }\end{array}$ & $\begin{array}{l}\text { Adherence to 4D } \\
\text { Self-report }\end{array}$ & $\begin{array}{l}\text { Non-adherence was } \\
\text { associated with age } \\
<50 \text { years (OR } 1.50,95 \% \\
\text { Cl } 1.08 \text { to } 2.09 ; p=0.015) \text {, } \\
\text { depression (OR } 1.07,95 \% \\
\text { Cl } 1.04 \text { to } 1.09 ; p<0.001 \text { ), } \\
\text { being on a complex } \\
\text { medication regimen (OR } \\
1.42,95 \% \text { Cl } 1.00 \text { to } 2.02 \text { : } \\
p=0.047 \text { ) and lower level of } \\
\text { social support (OR } 0.94 \\
0.92 \text { to } 0.96 ; p<0.001)\end{array}$ \\
\hline FDC & $\begin{array}{l}\text { Thom, } 2013 \\
\text { (India, } \\
\text { Europe) }\end{array}$ & High CV risk & RCT & 1698 & $\begin{array}{l}\text { Intervention=FDC (containing } \\
\text { either: } 75 \mathrm{mg} \text { aspirin, } 40 \mathrm{mg} \\
\text { simvastatin, } 10 \mathrm{mg} \text { lisinopril, } \\
\text { and } 50 \mathrm{mg} \text { atenolol or } 75 \mathrm{mg} \\
\text { aspirin, } 40 \mathrm{mg} \text { simvastatin, } \\
10 \mathrm{mg} \text { lisinopril and } 12.5 \mathrm{mg} \\
\text { hydrochlorothiazide) }\end{array}$ & $\begin{array}{l}\text { Adherence to } 4 \mathrm{D} \\
\text { at } 15 \text { months } \\
\text { Self-report }\end{array}$ & $\begin{array}{l}\text { FDC versus separate } \\
\text { medications: } R R 1.29,95 \% \\
C l 1.22 \text { to } 1.36 ; p<0.0001\end{array}$ \\
\hline \multirow[t]{3}{*}{ FDC } & $\begin{array}{l}\text { Castellano, } \\
2014 \\
\text { (Argentina, } \\
\text { Brazil, Italy, } \\
\text { Paraguay and } \\
\text { Spain) }\end{array}$ & $\begin{array}{l}\text { Aged }>40 \text { years } \\
\text { with AMI within } \\
\text { last } 2 \text { years. }\end{array}$ & $\mathrm{RCT}$ & 695 & $\begin{array}{l}\text { Intervention=FDC (containing } \\
\text { aspirin } 100 \mathrm{mg} \text {, simvastatin } \\
40 \mathrm{mg} \text { and ramipril } 2.5,5 \text { or } \\
10 \mathrm{mg} \text { ) }\end{array}$ & $\begin{array}{l}\text { Adherence at } \\
9 \text { months } \\
\text { Self-report and pill } \\
\text { count }\end{array}$ & $\begin{array}{l}\text { FDC versus separate } \\
\text { medications: } R R 1.24,95 \% \\
C l 1.06 \text { to } 1.47 ; p=0.009\end{array}$ \\
\hline & $\begin{array}{l}\text { Selak, } 2014 \\
\text { (New } \\
\text { Zealand) }{ }^{28}\end{array}$ & High CV risk & $\mathrm{RCT}$ & 233 & $\begin{array}{l}\text { Intervention=FDC (with two } \\
\text { versions available: aspirin } \\
75 \mathrm{mg} \text {, simvastatin } 40 \mathrm{mg} \text { and } \\
\text { lisinopril } 10 \mathrm{mg} \text { with either } \\
\text { atenolol } 50 \mathrm{mg} \text { or } \\
\text { hydrochlorothiazide } 12.5 \mathrm{mg} \text { ) }\end{array}$ & $\begin{array}{l}\text { Adherence to } 4 \mathrm{D} \\
\text { at } 12 \text { months } \\
\text { Self-report }\end{array}$ & $\begin{array}{l}\text { FDC versus separate } \\
\text { medications: } R R 1.50,95 \% \\
C l 1.25 \text { to } 1.82 ; p<0001\end{array}$ \\
\hline & $\begin{array}{l}\text { Patel, } 2015 \\
\text { (Australia, New } \\
\text { Zealand) }{ }^{26}\end{array}$ & High CV risk & $\mathrm{RCT}$ & 381 & $\begin{array}{l}\text { Intervention=FDC (containing } \\
\text { aspirin } 75 \mathrm{mg} \text {, simvastatin } \\
40 \mathrm{mg} \text {, lisinopril } 10 \mathrm{mg} \text { and } \\
\text { either atenolol } 50 \mathrm{mg} \text { or } \\
\text { hydrochlorothiazide } 12.5 \mathrm{mg} \text { ) }\end{array}$ & $\begin{array}{l}\text { Adherence to } 4 \mathrm{D} \\
\text { at } 18 \text { months } \\
\text { (median } \\
\text { follow-up) } \\
\text { Self-report }\end{array}$ & $\begin{array}{l}\text { FDC versus separate } \\
\text { medications: } R R 1.26,95 \% \\
C l 1.08 \text { to } 1.48 ; p<0001\end{array}$ \\
\hline
\end{tabular}




\begin{tabular}{|c|c|c|c|c|}
\hline $\begin{array}{l}\text { Barriers/ } \\
\text { facilitators }\end{array}$ & $\begin{array}{l}\text { Study (author, } \\
\text { year, setting) }\end{array}$ & Context & Study design & $\begin{array}{l}\text { Sample } \\
\text { size }\end{array}$ \\
\hline $\begin{array}{l}\text { Physician } \\
\text { education/ } \\
\text { training }\end{array}$ & $\begin{array}{l}\text { Ko, } 2005 \\
{\text { (Canada })^{18}}^{18}\end{array}$ & $\begin{array}{l}\text { AMI aged } \\
\geq 65 \text { years in } \\
\text { hospital }\end{array}$ & $\begin{array}{l}\text { Retrospective } \\
\text { cohort }\end{array}$ & 63301 \\
\hline
\end{tabular}

\section{Harats, $2005 \quad$ CHD in hospital Cross-sectional}

(Israel) $^{33}$

and prospective Cohort

Copayments for medical care

Winkelmayer, AMI in hospita 2007 (Austria) $^{34}$

\section{Ye, 2007} $(\text { USA })^{35}$

\section{CHD and}

hospital-initiated statin

Insurance and

prescription

\section{Choudhry,} 2011 (USA) $^{27}$

AMI in hospital

\section{RCT}

Retrospective

cohort

Retrospective cohort

Mathews, 2015 ACS in hospital $(\text { USA })^{38}$
Prospective cohort cost assistance

\section{Study details}

Evaluation on whether care by International medical graduates (IMGs) is a determinant of poor persistence and worse outcomes after AMI versus care by Canadian medical graduates (CMGs)

2994 Brief educational sessions with physicians to review National guidelines to ascertain physician's awareness

4105 The association between copayments and outpatient use of $\beta$ blockers, statins, and ACEl/ARB in Austrian M patients

5548 Databases containing inpatient admission, outpatient, enrollment and pharmacy claims from 1999 to 2003 to study associations with copayments

5855 Intervention=full prescription coverage by insurance-plan sponsor

Within the Treatment with Receptor Inhibitors: Longitudinal Assessment of Treatment Patterns and Events after Acute Coronary

Syndrome (TRANSLATE-ACS) study Adenosine Diphosphate

Relevant findings (95\%

Cls given where available and in italics when

Outcome $\mathbf{p}<0.05)$

Persistence to 4D Adjusted OR(Canadian/ at 90 days

Prescription refill IMG): aspirin $1.0095 \% \mathrm{C}$ (0.94 to 1.06); BB 1.01 (0.94 to 1.08); ACEI 1.07 (1.01 to 1.14$)$; statins 1.10 (1.01 to 1.20$)$

Intervention versus control:

Persistence to statins at 8 weeks Self-report

Adherence at 120 days Prescription refill rate

Adherence to statins at 1 year Prescription refill rate

$57 \%$ vs $45 \%$. $(p<0.001)$

Adherence (waived copayments versus copayment): OR 1.35; 95\% Cl 1.10 to 1.67 for ACEI/ $A R B$, OR $1.09 ; 0.89$ to 1.35) for $\beta$ blocker and OR $1.09 ; 0.89$ to 1.34 for statin Adherence (copayment $\geq$ US $\$ 20$ vs copayment $<$ US \$10): OR 0.42; $95 \% \mathrm{Cl} 0.36$ to 0.49

Adherence to 4D Full-coverage versus usual at 394 days (median

follow-up) coverage: OR $1.41,95 \% \mathrm{CI}$ 1.18 to $1.56 ; p<0.001$ for $4 D$ and $p<001$ for all Prescription refill individual drug classes rate

Persistence to 4D Non-persistence less likely at 6 months Self-report with private insurance (OR $0.85,95 \% \mathrm{Cl} 0.76$ to 0.95 ), prescription cost assistance (OR 0.63, 0.54 to 0.75 ), and clinic follow-up arranged predischarge (OR $0.89,0.80$ to 0.99 )

4D, secondary prevention drugs for CVD, namely, antiplatelets, $\beta$ blockers, angiotensin-converting enzyme inhibitor or angiotensin-receptor blockers and statins; ACEI, angiotensin-converting enzyme inhibitor; AMI, acute myocardial infarction; ACS, acute coronary syndrome; ARB, angiotensin-receptor blocker; CABG, coronary artery bypass graft; CHD, coronary heart disease; CVD, cardiovascular disease; FDC, fixed-dose combination therapy; GP, general practitioner; NSTEMI, non ST-elevation myocardial infarction; RCT, randomised controlled intervention trial; $\mathrm{RR}$, relative risk; TIA, transient ischaemic attack. 


\begin{tabular}{|c|c|c|c|c|c|c|c|c|c|}
\hline $\begin{array}{l}\text { Study } \\
\text { (author, year, } \\
\text { setting) }\end{array}$ & Context & Study design & $\begin{array}{l}\text { Random } \\
\text { sequence } \\
\text { generation } \\
\text { (selection } \\
\text { bias) } \\
\end{array}$ & $\begin{array}{l}\text { Allocation } \\
\text { concealment } \\
\text { (selection } \\
\text { bias) }\end{array}$ & $\begin{array}{l}\text { Blinding of } \\
\text { participants and } \\
\text { personnel } \\
\text { (performance } \\
\text { bias) }\end{array}$ & $\begin{array}{l}\text { Blinding of } \\
\text { outcome } \\
\text { assessment } \\
\text { (detection } \\
\text { bias) }\end{array}$ & $\begin{array}{l}\text { Incomplete } \\
\text { outcome } \\
\text { data } \\
\text { (attrition } \\
\text { bias) } \\
\end{array}$ & $\begin{array}{l}\text { Selective } \\
\text { reporting } \\
\text { (reporting } \\
\text { bias) } \\
\end{array}$ & $\begin{array}{l}\text { Risk of bias } \\
\text { assessment }\end{array}$ \\
\hline $\begin{array}{l}\text { O'Carroll, } \\
2013(\mathrm{UK})^{21}\end{array}$ & $\begin{array}{l}\text { First stroke/ } \\
\text { TIA }\end{array}$ & $\mathrm{RCT}$ & + & + & - & - & + & + & $\begin{array}{l}\text { High risk of } \\
\text { bias: } \\
\text { performance } \\
\text { and detection }\end{array}$ \\
\hline $\begin{array}{l}\text { Hornnes, } 2011 \\
\text { (Denmark) }^{22}\end{array}$ & $\begin{array}{l}\text { Acute } \\
\text { stroke/TIA }\end{array}$ & RCT & + & + & - & + & + & + & $\begin{array}{l}\text { High risk of } \\
\text { bias: } \\
\text { performance }\end{array}$ \\
\hline $\begin{array}{l}\text { McManus, } \\
2009 \text { (UK) }^{23}\end{array}$ & $\begin{array}{l}\text { Stroke in } \\
\text { hospital }\end{array}$ & $\mathrm{RCT}$ & + & + & + & + & + & + & $\begin{array}{l}\text { Low risk of } \\
\text { bias }\end{array}$ \\
\hline $\begin{array}{l}\text { Faulkner, } 2000 \\
\text { (USA) }^{17}\end{array}$ & CABG & $\mathrm{RCT}$ & + & - & - & - & + & + & $\begin{array}{l}\text { High risk of } \\
\text { bias: selection, } \\
\text { performance } \\
\text { and detection }\end{array}$ \\
\hline $\begin{array}{l}\text { Hohmann, } \\
2009 \\
\text { (Germany) }^{29}\end{array}$ & $\begin{array}{l}\text { Ischaemic } \\
\text { stroke/TIA in } \\
\text { hospital }\end{array}$ & $\begin{array}{l}\text { Non-randomised } \\
\text { controlled } \\
\text { intervention trial }\end{array}$ & - & - & - & - & + & + & $\begin{array}{l}\text { High risk of } \\
\text { bias: selection, } \\
\text { performance } \\
\text { and detection }\end{array}$ \\
\hline $\begin{array}{l}\text { Yilmaz, } 2005 \\
\text { (Turkey) }^{24}\end{array}$ & $\begin{array}{l}\text { Secondary } \\
\text { prevention in } \\
\text { hospital }\end{array}$ & $\mathrm{RCT}$ & $?$ & - & - & - & + & + & $\begin{array}{l}\text { High risk of } \\
\text { bias: selection, } \\
\text { performance } \\
\text { and detection }\end{array}$ \\
\hline $\begin{array}{l}\text { Johnston, } \\
2010 \text { (USA) }^{19}\end{array}$ & $\begin{array}{l}\text { Ischaemic } \\
\text { stroke in } \\
\text { hospital }\end{array}$ & $\mathrm{RCT}$ & + & + & - & - & + & + & $\begin{array}{l}\text { High risk of } \\
\text { bias: } \\
\text { performance } \\
\text { and detection }\end{array}$ \\
\hline $\begin{array}{l}\text { Thom, } 2013 \\
\text { (India, } \\
\text { Europe) }\end{array}$ & High CV risk & $\mathrm{RCT}$ & + & + & $?$ & $?$ & + & + & $\begin{array}{l}\text { Unclear risk of } \\
\text { bias: } \\
\text { performance } \\
\text { and detection }\end{array}$ \\
\hline $\begin{array}{l}\text { Castellano, } \\
2014 \\
\text { (Argentina, } \\
\text { Brazil, Italy, } \\
\text { Paraguay and } \\
\text { Spain) }^{25}\end{array}$ & $\begin{array}{l}\text { Aged } \\
>40 \text { years } \\
\text { with AMI } \\
\text { within last } \\
2 \text { years }\end{array}$ & $\mathrm{RCT}$ & + & + & $?$ & $?$ & + & + & $\begin{array}{l}\text { Unclear risk of } \\
\text { bias: } \\
\text { performance } \\
\text { and detection }\end{array}$ \\
\hline $\begin{array}{l}\text { Selak } 2014 \\
\text { (New } \\
\text { Zealand) }\end{array}$ & High CV risk & $\mathrm{RCT}$ & + & + & + & + & + & + & $\begin{array}{l}\text { Low risk of } \\
\text { bias }\end{array}$ \\
\hline
\end{tabular}




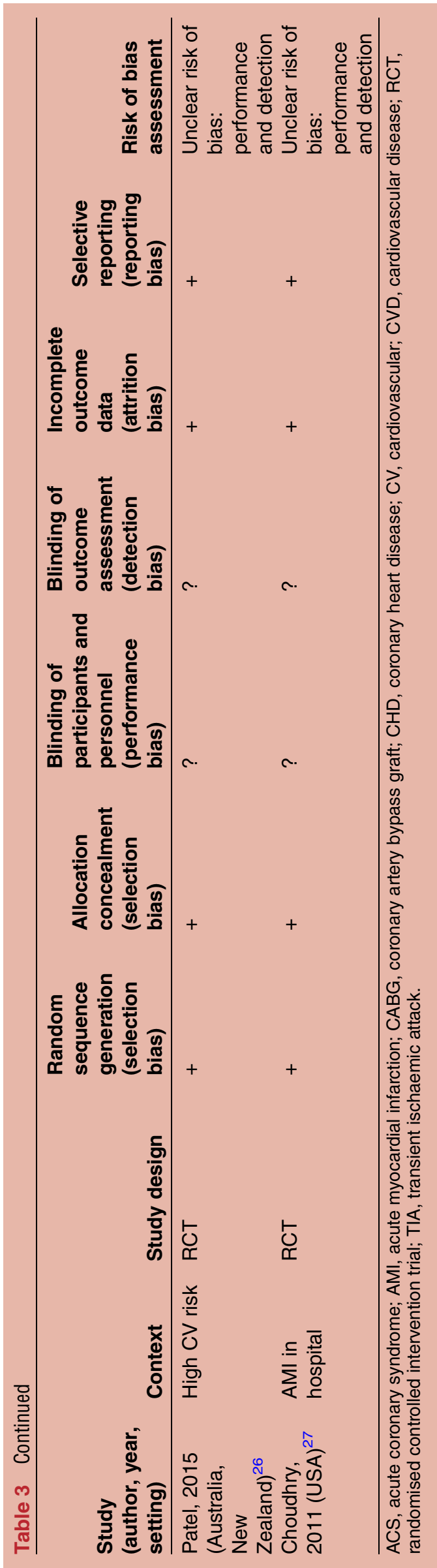

$(\mathrm{n}=4)^{20242628}$ and coronary heart disease (from stable $\mathrm{CHD}$, to acute myocardial infarction (AMI), to coronary artery bypass graft $(\mathrm{CABG}))(\mathrm{n}=14) .{ }^{17} 1825273133-40$

Thirteen studies included aspirin, $\beta$ blocker, ACEI/

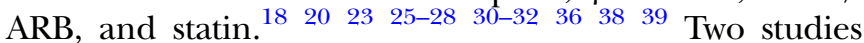
investigated antihypertensive medication, including $\beta$ blockers and ACEI/ARB, ${ }^{21}{ }^{22}$ and seven studies included only statins. ${ }^{17} 192433353740$ One study focused on antiplatelet drugs including aspirin. ${ }^{29}$ One study investigated $\beta$ blockers, statins and ACEI/ARB ${ }^{34}$ and one considered aspirin, ACEI and $\operatorname{statin}^{25}$ (see online supplementary appendix table 2c).

In 16 studies, indirect measures of adherence were employed: prescription refill rates (medication possession ratio (MPR), ${ }^{27} 35$ proportion of days covered $(\mathrm{PDC})^{37}$ and other measures), ${ }^{17} 193134$ electronic pill bottle count, ${ }^{21}$ manual pill count $^{25}$ and selfreport. $^{20} 222325262839$ Persistence was measured in 11 studies, by self-report ${ }^{23} 242930333638-40$ or prescription refill. ${ }^{18} 32$ Three studies considered persistence and adherence. $^{23} 3039$

Nineteen of 25 included studies were concerned with governance and delivery (table 1). ${ }^{17}$ 19-26 28-32 36373940 Only one study, based in Canada, considered human resource implications on adherence to medication in a retrospective cohort design. ${ }^{18}$ Only one study considered intellectual resources (impact of physician education on medication adherence) in patients admitted with CHD in Israel. ${ }^{33}$ Health financing was considered in four studies in the $\mathrm{USA}^{27} 3538$ and Austria, ${ }^{34}$ respectively. No studies examined the role of physical or social resources in medication adherence.

\section{Quality of included studies}

Only 2 of the 12 included trials were deemed to have low risk of bias, ${ }^{23} 28$ and 2 had an unclear degree of bias. ${ }^{20}{ }^{25}$ Remaining trials had high risk of bias in one or several domains, most commonly due to lack of blinding of participants, personnel or outcomes (table 3). Three included observational studies had low risk of bias in all domains $^{30-32}$ (table 4). Although meta-analysis was not undertaken, funnel plot asymmetry suggests possible publication bias (see online supplementary appendix figure 1).

\section{Barriers}

Complexity of treatment regimen

A cross-sectional study of individuals with myocardial infarction (MI) in the last 2 years in South America/ Europe showed that taking more than 10 pills $(p=0.047)$ and a complex regimen (eg, taking medications other than orally) $(\mathrm{p}=0.017)$ were associated with self-reported non-adherence. However, only a complex regimen was independently predictive of non-adherence (OR 1.42, 1.00 to $2.02 ; \mathrm{p}=0.047) .^{25}$ 


\section{Copayments for medical care}

Two retrospective cohort studies investigated the impact of copayments on adherence. Both studies had low risk of bias but high risk of confounding. Among 4105 patients with acute MI in Austria, ${ }^{34}$ those with waived copayments had higher persistence at 120 days for ACEI/ARB than those with copayments (OR 1.35, 95\% CI 1.10 to 1.67 ), but $\beta$ blocker (OR 1.09, 0.89 to 1.35 ) or statin use (OR 1.09, 0.89 to 1.34) did not differ between these groups. The second US study of CHD patients $^{35}$ found that compared with copayment $<\mathrm{US}$ $\$ 10$, copayment $\geq$ US $\$ 20$ was associated with lower persistence at 1 year for statins (OR 0.42; 0.36 to 0.49 , $\mathrm{p}<0.001)$.

\section{Insurance and prescription cost assistance}

A US-based prospective cohort study of 7955 MI patients in 216 hospitals showed that non-persistence to secondary prevention medications was less likely with private insurance (OR $0.85,95 \%$ CI 0.76 to 0.95 ) and prescription cost assistance (OR $0.63,95 \%$ CI 0.54 to 0.75$).{ }^{38} \mathrm{~A}$ US-based RCT included 5855 individuals post-MI, randomised to full or usual prescription coverage. ${ }^{27}$ Full adherence was higher with full prescription coverage for all medication classes (OR 1.41, 1.18 to $1.67 ; \mathrm{p}<0.001$ ). Increased adherence to all three medications for the patient subgroup undergoing CABG was found, post hoc (OR $1.67,95 \%$ CI 1.04 to $2.67 ; \mathrm{p}=0.03)$. $^{41}$

\section{Facilitators}

\section{Patient counselling}

Patient counselling was the most investigated facilitator of adherence/persistence (table 2). Only one study investigated impact of physician-led counselling following first stroke/transient ischaemic attack (TIA). This UK RCT included 62 individuals, with high risk of bias overall $^{21}$ (table 3). At 3 months, adherence to antihypertensive medication was higher in the intervention group for doses taken on schedule (ie, mean difference, $9.8 \%$; $95 \%$ CI (0.2 to 16.2$) ; \mathrm{p}=0.048)$.

Three studies considered nurse-led interventions in a total of 2738 individuals in Denmark, the USA/Canada and the UK, respectively, ${ }^{22} 23{ }^{39}$ with high risk of bias in the Danish study and high risk of confounding in the American study (table 3). The Danish study randomised stroke/TIA patients to four home visits with nurse-led counselling, finding no difference in self-reported adherence at 1 year between intervention and control groups (98\% vs 99\%, respectively, OR 0.88 , 95\% CI 0.54 to $1.44 ; \mathrm{p}=0.50) .^{22}$ The North American study assigned nurse case managers to patients with stable CHD over a 5 -year period, nested within a trial of percutaneous coronary intervention and optimal medical therapy versus optimal medical therapy alone. ${ }^{39}$ Persistence increased from baseline to 5 years across all drug categories, including all four drugs together (28\% to 53\%, OR 2.90, 95\% CI 2.44 to $3.43 ; \mathrm{p}<0.001)$. Self-reported adherence was not different between groups $(97 \%$ at 6 months and
$95 \%$ at 5 years, respectively). Lack of a control group limited study findings. In the UK-based RCT, ${ }^{23}$ patients who had stroke were randomised to nurse-led counselling sessions for 3 months. At 3 years, persistence and adherence were not different between groups (table 2).

Two studies investigated pharmacist-led counselling of patients: one RCT in the $\mathrm{USA}^{17}$ and one nonrandomised trial in Germany. ${ }^{29}$ The US trial included 30 patients following CABG, randomised to weekly pharmacist telephone calls for 3 months or usual care. Adherence to statins was higher in the intervention group versus the control group at 1 year (71\% vs $47 \%$; $\mathrm{p}<0.05)$ and at 2 years $(63 \%$ vs $39 \%)$ as measured by prescription refill, using the $\chi^{2}$ test. However, the effect was not statistically significant using ORs at 1 year (OR $4.00,0.88$ to $18.26 ; \mathrm{p}=0.07$ ) or 2 years (OR 4.13, 0.88 to 19.27; $\mathrm{p}=0.07)$. In the German trial, a hospital pharmacist delivered predischarge counselling to 255 stroke/ TIA patients was compared with usual care. At 1 year, persistence was $38.7 \%$ vs $32.7 \%$, OR $1.30,0.73$ to 2.31 ; $\mathrm{p}=0.37)$ for aspirin and $30.1 \%$ and $26.7 \% \quad(\mathrm{p}=0.60)$ for clopidogrel, in intervention versus control groups, respectively.

Two studies reported on the delivery of comprehensive health counselling in hospital-based patients. ${ }^{24} 36$ In a secondary prevention population of 202 individuals already on statins, a Turkish RCT $^{24}$ found that comprehensive counselling was associated with increased adherence compared with usual care $(62.7 \%$ vs $46 \%$; $\mathrm{OR}=1.98 ; \mathrm{p}=0.017$ ) after a median follow-up of 15 months. Bias due to contamination was likely as patients were already taking statins. In France, a prospective cohort study without the control group invited 660 patients 3 months after acute coronary syndrome for comprehensive risk factor management with a cardiologist. ${ }^{36}$ Persistence was $86 \%$ vs $98 \%$ for $\beta$ blocker/calcium antagonist, $88 \%$ vs $94 \%$ for cholesterol-lowering medication, $96 \%$ vs $100 \%$ for antiplatelet, $62 \%$ vs $82 \%$ for ACEI/ARB and $76 \%$ vs $92 \%$ for antiplatelet, $\beta$ blocker and cholesterol-lowering medications combined at follow-up (mean 20 months) and baseline, respectively (all $\mathrm{p}<0.0001)$. This study had high risk of bias across the domains of differential misclassification, nondifferential misclassification and confounding.

\section{Hospital-level quality improvement}

In this section, studies at the level of the hospital or a whole health system were considered. All four studies of hospital-level quality improvement were conducted in North America (three in the USA, ${ }^{19}{ }^{30}{ }^{40}$ one in Canada) ${ }^{31}$ with one RCT, two cohort studies and one case-control study (table 1 ). The case-control study and RCT had high risk of bias (tables 3 and 4). The RCT found that hospital-level assistance in the development and implementation of standardised stroke discharge orders was not associated with improved adherence over 12 months at the hospital level $(57.3 \%$ vs $62.9 \%$; OR $1.26,95 \%$ CI 0.70 to $2.30 ; \mathrm{p}=0.36)$, although there was 
Table 4 Risk of bias of included observational studies

\begin{tabular}{|c|c|c|c|c|c|c|c|}
\hline \multirow{2}{*}{$\begin{array}{l}\text { Study (author, year, } \\
\text { setting) }\end{array}$} & \multirow[b]{2}{*}{ Context } & \multirow[b]{2}{*}{ Study design } & \multicolumn{4}{|l|}{ Bias } & \multirow[b]{2}{*}{ Risk of bias assessment } \\
\hline & & & $\overline{\mathbf{S}}$ & DM & ND & C & \\
\hline $\begin{array}{l}\text { Maron, } 2010 \text { (USA and } \\
\text { Canada) }\end{array}$ & Stable CHD & Prospective cohort & Low & Low & Low & High & High risk of confounding \\
\hline Lafitte, 2009 (France) $^{36}$ & ACS in hospital & Prospective cohort & High & High & High & High & High risk of bias \\
\hline Bushnell, 2011 (USA) ${ }^{30}$ & $\begin{array}{l}\text { Ischaemic } \\
\text { stroke/TIA in } \\
\text { hospital }\end{array}$ & Retrospective cohort & Low & Low & Low & Low & Low risk of bias \\
\hline $\begin{array}{l}\text { Jackevicius, } 2008 \\
\text { (Canada) }^{31}\end{array}$ & AMI in hospital & Retrospective cohort & Low & Low & Low & Low & Low risk of bias \\
\hline $\begin{array}{l}\text { Khanderia, } 2005 \\
\text { (USA) }\end{array}$ & $\begin{array}{l}\text { CABG in } \\
\text { hospital }\end{array}$ & $\begin{array}{l}\text { Retrospective case- } \\
\text { control }\end{array}$ & High & Low & Low & High & High risk of bias \\
\hline $\begin{array}{l}\text { Glader, } 2010 \\
\text { (Sweden) }^{32}\end{array}$ & $\begin{array}{l}\text { Acute stroke in } \\
\text { hospital }\end{array}$ & Prospective cohort & Low & Low & Low & Low & Low risk of bias \\
\hline O'Brien, 2015 (USA) ${ }^{37}$ & $\begin{array}{l}\text { NSTEMI in } \\
\text { hospital }\end{array}$ & Retrospective cohort & Low & Low & Low & High & $\begin{array}{l}\text { Low risk of bias, but high } \\
\text { risk of confounding }\end{array}$ \\
\hline $\begin{array}{l}\text { Castellano, } 2014 \\
\text { (Argentina, Brazil, Italy, } \\
\text { Paraguay and Spain) }\end{array}$ & $\begin{array}{l}\text { Aged }>40 \text { years } \\
\text { with AMI within } \\
\text { last } 2 \text { years }\end{array}$ & $\begin{array}{l}\text { Cross-sectional } \\
\text { study }\end{array}$ & High & Low & Low & High & $\begin{array}{l}\text { High risk of bias- } \\
\text { selection bias and } \\
\text { confounding }\end{array}$ \\
\hline Ko, 2005 (Canada) $^{18}$ & $\begin{array}{l}\text { AMl aged } \\
\geq 65 \text { years in } \\
\text { hospital }\end{array}$ & Retrospective cohort & Low & Low & Low & Unclear & $\begin{array}{l}\text { Low risk of bias but } \\
\text { unclear risk of } \\
\text { confounding }\end{array}$ \\
\hline Harats, 2005 (Israel) $^{33}$ & CHD in hospital & $\begin{array}{l}\text { Cross-sectional then } \\
\text { prospective cohort }\end{array}$ & Low & High & Low & High & $\begin{array}{l}\text { High risk of bias due to } \\
\text { differential } \\
\text { misclassification and } \\
\text { confounding }\end{array}$ \\
\hline $\begin{array}{l}\text { Winkelmayer, } 2007 \\
\text { (Austria) }\end{array}$ & AMI in hospital & Retrospective cohort & Low & Low & Low & High & $\begin{array}{l}\text { Low risk of bias but high } \\
\text { risk of confounding }\end{array}$ \\
\hline Ye, 2007 (USA) $)^{35}$ & $\begin{array}{l}\text { CHD and } \\
\text { initiated statin in } \\
\text { hospital }\end{array}$ & Retrospective cohort & Low & Low & Low & High & $\begin{array}{l}\text { Low risk of bias but high } \\
\text { risk of confounding }\end{array}$ \\
\hline $\begin{array}{l}\text { Mathews, } 2015 \\
(\text { USA) })^{38}\end{array}$ & ACS in hospital & Prospective cohort & Low & Low & Low & High & $\begin{array}{l}\text { Low risk of bias but high } \\
\text { risk of confounding }\end{array}$ \\
\hline
\end{tabular}

improvement in adherence to statins at the individual level (OR 1.29, 1.04 to $1.60 ; \mathrm{p}=0.02) .{ }^{19}$

One retrospective cohort study in acute MI patients in Canada $^{31}$ showed that predischarge medication counselling $(76.0 \%$ vs $64.8 \%$; OR $1.61,1.26$ to $2.04 ; \mathrm{p}=0.0001)$ and having a cardiologist (vs general practitioner) responsible for patient care were associated with adherence at 120 days postdischarge $(34.5 \%$ vs $25.4 \%$; OR $1.80,1.34$ to $2.43 ; \mathrm{p}=0.0001)$, with no association with treatment at a teaching hospital $(14.8 \%$ vs $11.8 \%$; OR $1.35,0.93$ to $1.97 ; \mathrm{p}=0.11)$. In a retrospective cohort study of stroke/TIA patients in the USA, ${ }^{30} 12$-month persistence with secondary prevention medications was associated with fewer medications $(\mathrm{OR}=1.04,1.02$ to 1.06 , $\mathrm{p}<0.001$ per one medication decrease); in-patient rehabilitation $(13.4 \%$ vs $21.6 \%$; $\mathrm{OR}=0.57,0.43$ to 0.76 , $\mathrm{p}<0.001)$; primary care follow-up $(92.1 \%$ vs $88.4 \%$; $\mathrm{OR}=1.47, \quad 1.05$ to $2.07, \mathrm{p}=.0 .027)$ and neurology follow-up $(43.3 \%$ vs $35.0 \% ; \quad \mathrm{OR}=1.20,1.03$ to 1.41 , $\mathrm{p}=0.023)$. Fewer medications $(\mathrm{OR}=1.08,1.04$ to 1.11 , $\mathrm{p}<0.001$ per 1 decrease) and primary care follow-up
$(\mathrm{OR}=1.72,1.12$ to $2.52, \mathrm{p}=0.006)$ were associated with persistence and adherence. A retrospective case-control study of CABG patients found that a physician education protocol was not associated with improved adherence to statins at 6 months. ${ }^{40}$

Site of care and home circumstances of patients

A Swedish prospective cohort study of patients who had stroke $^{32}$ found that institutional living was correlated with persistence of all secondary prevention medications $(\mathrm{p}=0.001)$. Stroke unit care was associated with persistence for statins $(p=0.007)$, and next-of-kin support was associated with persistence for antihypertensives $(\mathrm{p}=0.001)$.

\section{Generic versus branded medication prescription}

A US retrospective, hospital-based cohort study of individuals post-NSTEMI (non-ST elevation myocardial infarction) investigated 1-year statin adherence by prescription of generic versus branded medications. ${ }^{37}$ Although patient copayment amounts were higher for brand 
versus generic statins (median=US\$25 vs US $\$ 5$, $\mathrm{p}<0.001)$, there was no statistically significant difference in adherence over 1 year $(71.5 \%$ vs $68.9 \%$, unadjusted OR $1.15,95 \%$ CI 0.96 to $1.37 ; \mathrm{p}=0.97$ ).

\section{Fixed-dose combination therapy}

Four RCTs examined fixed-dose combination therapy (FDC). ${ }^{20} 252628$ One RCT in Europe and India in 1698 individuals with established CVD demonstrated that FDC (either aspirin/simvastatin/lisinopril/atenolol or hydrochlorothiazide) was associated with higher self-reported adherence than routine therapy (RR 1.29, 95\% CI 1.22 to $1.36 ; \mathrm{p}<0.0001) .{ }^{20}$ In 695 individuals in Argentina, Brazil, Italy, Paraguay and Spain, FDC (containing aspirin/simvastatin/ramipril) was compared with the three drugs given separately in individuals with MI in the last 2 years. ${ }^{25}$ Adherence (by self-report and pill count) was higher with FDC (RR 1.24, 95\% CI 1.06 to 1.47; $\mathrm{p}=0.009$ ). A trial in Australia/New Zealand compared FDC (containing aspirin/simvastatin/lisinopril/ atenolol or hydrochlorothiazide) with usual care in 381 individuals with established CVD. Self-reported adherence was higher with FDC (RR 1.49; $95 \%$ CI 1.30 to 1.72; $\mathrm{p}<0.0001) .{ }^{26} \mathrm{~A}$ further trial in New Zealand compared FDC (containing aspirin/simvastatin/lisinopril/ atenolol or hydrochlorothiazide) with usual care in 233 individuals with established CVD. ${ }^{28}$ Self-reported adherence was greater with FDC (RR 1.75, 95\% CI 1.52 to 2.03, $\mathrm{p}<0.001)$. Of the four trials, only the New Zealand RCT had low risk of bias with the other three having unclear risk of performance and detection biases (table 3).

\section{Physician education/training}

Among 63301 acute MI patients, there was no significant difference in medication persistence at 90 days between patients treated by non-Canadian versus Canadian graduates for aspirin (OR 1.00, 0.94 to 1.06) or $\beta$ blocker (OR 1.01, 0.94 to 1.08). There was increased persistence in the non-Canadian medical graduate-treated group for ACEI (OR 1.07, 1.01 to 1.14) and statins (OR 1.10, 1.01 to 1.20), compared with patients treated by Canadian medical graduates. ${ }^{18}$ The relevance of these findings is reduced by the low ORs, a plausible mechanism for differential effect on different drugs and the unclear risk of confounding. In a hospitalbased study of CHD patients in Israel, an educational programme for physicians increased self-reported persistence to statins at 8 weeks follow-up $(57 \%$ vs $45 \%$ at follow-up; $\mathrm{p}<0.001)$. However, there was high risk of differential misclassification bias and confounding.

\section{Persistence and adherence}

Adherence was assessed across studies from 3 months to 5 years (see online supplementary appendix table $1 \mathrm{a}$ ). The three studies with follow-up $\geq 2$ years did not show increased adherence. ${ }^{17} 2339$ FDC consistently increased adherence across four RCTs with follow-up at between 9 and 18 months. ${ }^{20} 252628$ Copayments, insurance and prescription coverage were all associated with increased adherence from 120 days to 1 year. ${ }^{27} 3435$

Two RCTs ${ }^{23}{ }^{24}$ and one non-randomised trial ${ }^{29}$ had persistence outcomes, and only one RCT showed a positive impact of counselling on statin persistence at 15 months median follow-up ${ }^{24}$ (see online supplementary table $1 \mathrm{~b}$ ). The only trial considering persistence and adherence did not show effect on these outcomes. ${ }^{23}$ Nurse-led case management was associated with increased persistence at 5 years but not increased adherence. $^{39}$

\section{Different medication classes}

Of the studies considering all four classes of secondary prevention medications, FDC and prescription coverage were supported by RCT evidence. ${ }^{20} 26-28$ The two RCTs of counselling on antihypertensive therapy had conflicting findings. ${ }^{21}{ }^{22}$ Overall, there were insufficient numbers of studies considering individual classes of medications and lack of consistency of findings within the existing studies to suggest varying effects of interventions across different classes of medications.

\section{DISCUSSION}

The World Heart Federation's '25 by 25' strategy to tackle the CVD epidemic and specifically its recent roadmap for secondary prevention give context to our four key findings. ${ }^{12}$ First, at health systems level, there is lack of high-quality evidence on barriers and facilitators (especially from low and low-middle income countries) to adherence and persistence for the most wellestablished therapies available for secondary prevention of CVD. The only barriers with available data were complexity of treatment regimen and health financing. Several potential facilitators have been considered but not in a coordinated manner for adherence and persistence, or different classes of drugs. Second, FDC therapy is associated with increased adherence. Third, reduced copayments and full prescription coverage were associated with increased adherence and persistence. Finally, counselling of patients whether by doctor, nurse or pharmacist can result in improved adherence to secondary prevention CVD medications.

There is high-quality evidence to support use of aspirin, ACEIs/ARBs, $\beta$ blockers and statins in secondary prevention of CVD. ${ }^{9} 42$ However, a recent analysis of Cochrane reviews and RCTs in non-communicable diseases (including CVD) showed that almost $90 \%$ of trials and over $80 \%$ of participants were from high-income countries, ${ }^{43}$ and our analysis further highlights the sparse data for policymakers to make evidence-based changes to improve adherence to secondary prevention medications for CVD, particularly in low-income settings. The overall quality of evidence is low by objective criteria, ${ }^{44}$ due to lack of directness of evidence, heterogeneity across studies and only $12(48 \%)$ of the 25 included studies being RCTs. There were no studies in low-income 
countries and only one study in an upper middle-income country, and two studies included a lower middle-income country. ${ }^{20}{ }^{24}$ Moreover, there were neither quantitative studies regarding physical resources and social resources nor qualitative studies of adherence to secondary CVD prevention medications. As well as limited study numbers, heterogeneity in study design, study populations, study quality, health system arrangements and outcomes make meta-analysis and synthesis of the available data extremely challenging. Given the lack of large-scale and generalisable data to support changes in health systems to improve adherence to medications for CVD, policymakers can take advantage of context-specific information, including quantitative data. We were unable to find any qualitative studies meeting our inclusion criteria, but this particular study design may be informative in understanding local context for patients, healthcare providers and policymakers.

The imbalance between efficacy research (eg, pharmacologic trials with 'hard' clinical outcomes) and policyoriented implementation research (eg, adherence) is well documented in CVD and other diseases. ${ }^{45-47}$ The World Heart Federation's Roadmap has emphasised the "'treatment effectiveness cascade' from the cardiovascular event to the long-term adherence with priority interventions". ${ }^{12}$ This systematic review illustrates that, particularly in low-income settings, the understanding of health system barriers and facilitators is poor from a research perspective, and that practical research and pragmatic solutions are urgently required, if the World Heart Federation's goals of reducing premature CVD mortality by at least $25 \%$ by 2025 are to be fulfilled.

Poor adherence to prescribed therapy has been estimated to account for at least 9\% of CVD events in Europe alone ${ }^{48}$ and optimal adherence is associated with better outcomes in CVD. ${ }^{49}$ Importantly, FDC therapy has been shown to improve adherence in other diseases including hypertension ${ }^{50}$ and HIV/AIDS. ${ }^{51}$ FDC therapy may improve adherence in CVD secondary prevention on the basis of four trials, ${ }^{20} 252628$ but the results of several other trials are awaited and there is still uncertainty whether FDC therapy influences long-term outcomes. ${ }^{25} 52$ There are concerns regarding the exact combination and dose of medications in FDC therapy, as shown by variations in combinations used in the trials included in our review, but this strategy may standardise supply and use of the component medications, ${ }^{53}$ leading to calls for its inclusion in the WHO essential medicines list. $^{54}$

The secondary prevention medications investigated in this review are off-patent and likely to be the most widely available medications for treatment of CVD. However, we show that lower or no copayment was still associated with higher adherence to therapy in CVD, as illustrated by previous analyses. ${ }^{55} 56$ These findings echo the conclusions of prior analyses of health system financing in other disease areas, ${ }^{57}$ suggesting as with FDC therapy, that in the field of adherence to CVD drug therapy, there is scope for research, practice and policy to cross disease boundaries, especially in low-income countries. Universal healthcare coverage has been emphasised in the sustainable development goals, ${ }^{58}$ offering opportunities and challenges to embed full prescription coverage for secondary prevention for CVD in cross-sectoral approaches, which research and global health player must embrace.

Global shortage of health workers is a threat to provision of healthcare including CVD prevention. ${ }^{59}$ The finding that counselling of patients, regardless of the profession of the health worker, can result in improved adherence to secondary prevention CVD medications has significance in the context of international debate about universal health coverage and task shifting of health workers. Unlike other disease areas, the implementation of task-shifting strategies for CVD is lacking in an evidence base in low-income settings, ${ }^{60}$ and this should be a focus of future research in secondary CVD prevention and improvement strategies for medication adherence.

\section{Limitations}

This study, like any systematic review, was limited by inclusion criteria, and we restricted our study to secondary prevention medications for CVD. The initiation and use of medications was not studied, only studies of adherence and persistence were included and therefore not all barriers and facilitators to appropriate medication use were investigated in this analysis. Only published literature was considered. We were able to include only 25 studies in our systematic review as already discussed. The studies could not be weighted by relative relevance of each of these results as they are context limited. However, the interventions recommended by this review are unlikely to be dependent on context. Meta-analysis was not possible due to heterogeneity in several respects. We did not assess conflict of interest. Conclusions with respect to particular barriers and facilitators, drug class and persistence versus adherence were limited by the number of studies available. Generalisability of findings is questionable, suggesting that a common methodology for studies of medication adherence (and persistence) in CVD and other diseases is required, as well as urgent need for research in lowincome settings.

\section{CONCLUSIONS}

To the best of our knowledge, this is the first systematic review of health system influences on adherence to evidence-based secondary prevention therapies in CVD. Lack of generalisable, high-quality evidence to inform policy to improve adherence highlights the pressing need for research, particularly in low-income countries. Full prescription coverage and reduced copayments, FDC therapy and patient counselling are supported by existing literature as strategies to improve adherence 
and are priorities for further research before implementation. Standardised definitions of and approaches to adherence and persistence are also required in consensus guidelines for management of CVD.

\section{Author affiliations}

${ }^{1}$ Farr Institute of Health Informatics Research, University College London, London, UK

${ }^{2}$ Public Health Foundation of India, New Delhi, India

${ }^{3}$ Population Health Research Institute, McMaster University, Hamilton, Ontario, Canada

${ }^{4}$ WHO Bangladesh, Dhaka, Bangladesh

${ }^{5}$ Pontifícia Universidade Católica do Paraná (PUCPR), Curitiba, Brazil

${ }^{6}$ Instituto de Nutrición y Tecnología de los Alimentos, Asociación

Kausasunchis-ADEK Perú, Lima, Peru

${ }^{7}$ Centro Cardiologico Monzino, IRCCS, Milan, Italy

Twitter Follow Amitava Banerjee at @amibanerjee1

Acknowledgements The authors acknowledge the help and support of the World Heart Federation Emerging Leaders Programme and particularly Mark Huffman and Darwin Labarthe who commented on earlier drafts of the manuscript.

Contributors All coauthors contributed to the idea and methodology of the systematic review, as well as the search, study selection and data extraction. $A B$ conducted the initial analyses and wrote the first draft of the manuscript. All coauthors contributed to interpretation of data and the final version of the manuscript.

Funding World Heart Federation Emerging Leaders Programme.

Competing interests None declared.

Provenance and peer review Not commissioned; externally peer reviewed.

Data sharing statement Any requests for data sharing should be made to the corresponding author.

Open Access This is an Open Access article distributed in accordance with the Creative Commons Attribution Non Commercial (CC BY-NC 4.0) license, which permits others to distribute, remix, adapt, build upon this work noncommercially, and license their derivative works on different terms, provided the original work is properly cited and the use is non-commercial. See: http:// creativecommons.org/licenses/by-nc/4.0/

\section{REFERENCES}

1. Mensah GA, Moran AE, Roth GA, et al. The global burden of cardiovascular diseases, 1990-2010. Glob Heart 2014;9:183-4.

2. Cook C, Cole G, Asaria $P$, et al. The annual global economic burden of heart failure. Int J Cardiol 2014;171:368-76.

3. Muka T, Imo D, Jaspers $L$, et al. The global impact of non-communicable diseases on healthcare spending and national income: a systematic review. Eur J Epidemiol 2015;30:251-77.

4. Kankeu HT, Saksena P, Xu K, et al. The financial burden from non-communicable diseases in low- and middle-income countries: a literature review. Health Res Policy Syst 2013;11:31.

5. Beaglehole R, Bonita R, Lancet NCD Action Group. Tackling NCDs: a different approach is needed. Lancet 2012;379:1873; author reply-4.

6. Maher A, Sridhar D. Political priority in the global fight against non-communicable diseases. J Glob Health 2012;2:020403.

7. Joshi R, Jan $\mathrm{S}, \mathrm{Wu} \mathrm{Y}$, et al. Global inequalities in access to cardiovascular health care: our greatest challenge. J Am Coll Cardiol 2008:52:1817-25.

8. Fuster V. Global burden of cardiovascular disease: time to implement feasible strategies and to monitor results. J Am Coll Cardiol 2014;64:520-2.

9. Wald NJ, Law MR. A strategy to reduce cardiovascular disease by more than 80\%. BMJ 2003;326:1419.

10. Yusuf S, Islam S, Chow CK, et al. Use of secondary prevention drugs for cardiovascular disease in the community in high-income, middle-income, and low-income countries (the PURE Study): a prospective epidemiological survey. Lancet 2011;378:1231-43.
11. Kotseva K, Wood D, De Bacquer D, et al. EUROASPIRE IV: a European Society of Cardiology survey on the lifestyle, risk factor and therapeutic management of coronary patients from 24 European countries. Eur J Prev Cardiol 2016;23:636-48.

12. Perel $P$, Avezum A, Huffman M, et al. Reducing premature cardiovascular morbidity and mortality in people with atherosclerotic vascular disease: the world heart federation roadmap for secondary prevention of cardiovascular disease. Glob Heart 2015;10:99-110.

13. Yusuf S, Rangarajan S, Teo K, et al. Cardiovascular risk and events in 17 low-, middle-, and high-income countries. N Engl J Med 2014;371:818-27.

14. Maimaris W, Paty J, Perel P, et al. The influence of health systems on hypertension awareness, treatment, and control: a systematic literature review. PLoS Med 2013;10:e1001490.

15. Cramer JA, Roy A, Burrell A, et al. Medication compliance and persistence: terminology and definitions. Value Health 2008:11:44-7.

16. Liberati A, Altman DG, Tetzlaff $\mathrm{J}$, et al. The PRISMA statement for reporting systematic reviews and meta-analyses of studies that evaluate healthcare interventions: explanation and elaboration. $B M J$ 2009;339:b2700.

17. Faulkner MA, Wadibia EC, Lucas BD, et al. Impact of pharmacy counseling on compliance and effectiveness of combination lipid-lowering therapy in patients undergoing coronary artery revascularization: a randomized, controlled trial. Pharmacotherapy 2000;20:410-6.

18. Ko DT, Austin PC, Chan BT, et al. Quality of care of international and Canadian medical graduates in acute myocardial infarction. Arch Intern Med 2005;165:458-63.

19. Johnston SC, Sidney S, Hills NK, et al. Standardized discharge orders after stroke: results of the quality improvement in stroke prevention (QUISP) cluster randomized trial. Ann Neurol 2010;67:579-89.

20. Thom S, Poulter N, Field J, et al. Effects of a fixed-dose combination strategy on adherence and risk factors in patients with or at high risk of CVD: the UMPIRE randomized clinical trial. JAMA 2013;310:918-29.

21. O'Carroll RE, Chambers JA, Dennis M, et al. Improving adherence to medication in stroke survivors: a pilot randomised controlled trial. Ann Behav Med 2013;46:358-68.

22. Hornnes N, Larsen K, Boysen G. Blood pressure 1 year after stroke: the need to optimize secondary prevention. J Stroke Cerebrovasc Dis 2011;20:16-23.

23. McManus JA, Craig A, McAlpine $C$, et al. Does behaviour modification affect post-stroke risk factor control? Three-year followup of a randomized controlled trial. Clin Rehabil 2009;23:99-105.

24. Yilmaz MB, Pinar M, Naharci I, et al. Being well-informed about statin is associated with continuous adherence and reaching targets. Cardiovasc Drugs Ther 2005;19:437-40.

25. Castellano JM, Sanz G, Peñalvo JL, et al. A polypill strategy to improve adherence: results from the FOCUS project. J Am Coll Cardiol 2014:64:2071-82.

26. Patel A, Cass A, Peiris D, et al. Kanyini Guidelines Adherence with the Polypill (Kanyini GAP) Collaboration. A pragmatic randomized trial of a polypill-based strategy to improve use of indicated preventive treatments in people at high cardiovascular disease risk. Eur J Prev Cardiol 2015;22:920-30.

27. Choudhry NK, Avorn J, Glynn RJ, et al. Full coverage for preventive medications after myocardial infarction. $N$ Engl J Med 2011;365:2088-97.

28. Selak V, Elley CR, Bullen C, et al. Effect of fixed dose combination treatment on adherence and risk factor control among patients at high risk of cardiovascular disease: randomised controlled trial in primary care. BMJ 2014;348:g3318.

29. Hohmann C, Klotz JM, Radziwill R, et al. Pharmaceutical care for patients with ischemic stroke: improving the patients quality of life. Pharm World Sci 2009;31:550-8

30. Bushnell CD, Olson DM, Zhao X, et al. Secondary preventive medication persistence and adherence 1 year after stroke. Neurology 2011;77:1182-90.

31. Jackevicius CA, Li P, Tu JV. Prevalence, predictors, and outcomes of primary nonadherence after acute myocardial infarction. Circulation 2008;117:1028-36.

32. Glader EL, Sjölander M, Eriksson M, et al. Persistent use of secondary preventive drugs declines rapidly during the first 2 years after stroke. Stroke 2010;41:397-401.

33. Harats D, Leibovitz E, Maislos M, et al. Cardiovascular risk assessment and treatment to target low density lipoprotein levels in hospitalized ischemic heart disease patients: results of the HOLEM study. Isr Med Assoc J 2005;7:355-9. 
34. Winkelmayer WC, Bucsics AE, Schautzer A, et al. Use of recommended medications after myocardial infarction in Austria. Eur $J$ Epidemiol 2008;23:153-62.

35. Ye X, Gross CR, Schommer J, et al. Association between copayment and adherence to statin treatment initiated after coronary heart disease hospitalization: a longitudinal, retrospective, cohort study. Clin Ther 2007;29:2748-57.

36. Lafitte $M$, Pradeau V, Leroux L, et al. Efficacy over time of a short overall atherosclerosis management programme on the reduction of cardiovascular risk in patients after an acute coronary syndrome. Arch Cardiovasc Dis 2009;102:51-8.

37. O'Brien EC McCoy LA, Thomas L, et al. Patient adherence to generic versus brand statin therapy after acute myocardial infarction: insights from the Can Rapid Stratification of Unstable Angina Patients Suppress Adverse Outcomes with Early Implementation of the American College of Cardiology/American Heart Association Guidelines Registry. Am Heart $J$ 2015;170:55-61.

38. Mathews R, Wang TY, Honeycutt E, et al. TRANSLATE-ACS Study Investigators. Persistence with secondary prevention medications after acute myocardial infarction: insights from the

TRANSLATE-ACS study. Am Heart J 2015;170:62-9.

39. Maron DJ, Boden WE, O'Rourke RA, et al. Intensive multifactorial intervention for stable coronary artery disease: optimal medical therapy in the COURAGE (Clinical Outcomes Utilizing Revascularization and Aggressive Drug Evaluation) trial. J Am Coll Cardiol 2010;55:1348-58.

40. Khanderia U, Townsend KA, Eagle K, et al. Statin initiation following coronary artery bypass grafting: outcome of a hospital discharge protocol. Chest 2005;127:455-63.

41. Kulik A, Desai NR, Shrank WH, et al. Full prescription coverage versus usual prescription coverage after coronary artery bypass graft surgery: analysis from the post-myocardial infarction Free Rx Event and Economic Evaluation (FREEE) randomized trial. Circulation 2013;128:S219-25.

42. Wiley B, Fuster V. The concept of the polypill in the prevention of cardiovascular disease. Ann Glob Health 2014;80:24-34.

43. Heneghan C, Blacklock C, Perera R, et al. Evidence for non-communicable diseases: analysis of Cochrane reviews and randomised trials by World Bank classification. BMJ Open 2013;3: pii:e003298

44. Atkins D, Best D, Briss PA, et al. Grading quality of evidence and strength of recommendations. BMJ 2004;328:1490.

45. Amsterdam EA, Laslett L, Diercks D, et al. Reducing the knowledge-practice gap in the management of patients with cardiovascular disease. Prev Cardiol 2002;5:12-5.

46. Rebello TJ, Marques A, Gureje O, et al. Innovative strategies for closing the mental health treatment gap globally. Curr Opin Psychiatry 2014;27:308-14.
47. Sturke R, Harmston C, Simonds RJ, et al. A multi-disciplinary approach to implementation science: the NIH-PEPFAR PMTCT implementation science alliance. J Acquir Immune Defic Syndr 2014:67(Suppl 2):S163-7.

48. Chowdhury R, Khan H, Heydon E, et al. Adherence to cardiovascular therapy: a meta-analysis of prevalence and clinical consequences. Eur Heart J 2013;34:2940-8.

49. Ho PM, Bryson CL, Rumsfeld JS. Medication adherence: its importance in cardiovascular outcomes. Circulation 2009;119:3028-35.

50. Gupta AK, Arshad S, Poulter NR. Compliance, safety, and effectiveness of fixed-dose combinations of antihypertensive agents: a meta-analysis. Hypertension 2010;55:399-407.

51. Ramjan R, Calmy A, Vitoria M, et al. Systematic review and meta-analysis: patient and programme impact of fixed-dose combination antiretroviral therapy. Trop Med Int Health 2014;19:501-13.

52. de Cates AN, Farr MR, Wright N, et al. Fixed-dose combination therapy for the prevention of cardiovascular disease. Cochrane Database Syst Rev 2014;4:CD009868.

53. Yusuf S, Attaran A, Bosch J, et al. Combination pharmacotherapy to prevent cardiovascular disease: present status and challenges. Eur Heart J 2014;35:353-64.

54. Huffman MD, Yusuf S. Polypills: essential medicines for cardiovascular disease secondary prevention? J Am Coll Cardiol 2014;63:1368-70.

55. Lemstra M, Blackburn D, Crawley A, et al. Proportion and risk indicators of nonadherence to statin therapy: a meta-analysis. Can $J$ Cardiol 2012;28:574-80.

56. Sanfélix-Gimeno G, Peiró S, Ferreros I, et al. Adherence to evidence-based therapies after acute coronary syndrome: a retrospective population-based cohort study linking hospital, outpatient, and pharmacy health information systems in Valencia, Spain. J Manag Care Pharm 2013;19:247-57.

57. Boyer S, Clerc I, Bonono CR, et al. Non-adherence to antiretroviral treatment and unplanned treatment interruption among people living with HIV/AIDS in Cameroon: Individual and healthcare supply-related factors. Soc Sci Med 2011;72:1383-92.

58. Schmidt H, Gostin LO, Emanuel EJ. Public health, universal health coverage, and sustainable development goals: can they coexist? Lancet 2015;386:928-30.

59. Tsolekile LP, Abrahams-Gessel S, Puoane T. Healthcare professional shortage and task-shifting to prevent cardiovascular disease: implications for low- and middle-income countries. Curr Cardiol Rep 2015;17:115

60. Ogedegbe G, Gyamfi J, Plange-Rhule J, et al. Task shifting interventions for cardiovascular risk reduction in low-income and middle-income countries: a systematic review of randomised controlled trials. BMJ Open 2014;4:e005983. 